\title{
A Carcinoid Tumor of The Esophagus Treated with Endoscopic Ablation Therapy
}

\author{
Na Rae Kim, ${ }^{1}$ Yoon Jae Kim, ${ }^{2}$ In Cheol Hwang ${ }^{3}$ \\ 'Department of Pathology, Gachon University Gil Medical Center, Namdong-gu, Republic of Korea, ${ }^{2}$ Division of \\ Gastroenterology, Department of Internal Medicine, Gachon University Gil Medical Center, Namdong-gu, Republic \\ of Korea, ${ }^{3}$ Department of Family Medicine, Gachon University Gil Medical Center, Namdong-gu, Republic of Korea.
}

\section{ABSTRACT}

Esophageal carcinoids are exceedingly rare and only a few limited cases have been reported. Because knowledge about esophageal carcinoid is based primarily on case reports, the treatment of choice has not been settled. We report here on a localized carcinoid tumor in the esophagogastric junction. As our initial biopsy was nearly equal for the excision, we only performed coagulation ablation therapy instead of a wide excision which is traditionally recommended. The patient is free of recurrence so far during 50-month follow-up. Our report suggests that esophageal carcinoids may be not particularly associated with a poor prognosis.

Keywords: carcinoid tumor; esophagus; argon plasma coagulation.

\section{INTRODUCTION}

Carcinoid tumors (CTs) are neuroendocrine tumors that are thought to arise from amine precursor uptake and decarboxylation cells. ${ }^{1}$ Because the distribution of these cells in the esophagus is sparse compared to that in other area of the gastrointestinal tract, esophageal CTs are exceedingly rare. ${ }^{2}$ In addition, CT in esophagus is known to be found at advanced stage. ${ }^{3,4}$ Because of their malignant potential, the recommended treatment of esophageal CT has traditionally been resection regardless of their stage. Here we report a successful management of localized esophageal CT using endoscopic ablation.

\section{CASE REPORT}

A 56-year-old man visited for stomach cancer screening: most Korean people age 40 years and older undergo biennial gastric cancer screening with either upper gastrointestinal series or endoscopy through the National Health Insurance Corporation. Gastroscopy revealed a $0.5 \mathrm{~cm}$-sized erosive mucosal lesion with a smooth surface in the esophagogastric junction (Figure
1A), and a biopsy was then performed. Endoscopic ultrasonography demonstrated that the lesion was a 0.6 $\mathrm{cm}$-sized demarcated hypoechoic mass confined to the submucosal layer (Figure 1B). Pathologically, trabecular and anastomosing ribbon-like nests intermixed with solid nests were scattered in the lamina propria (Figure 1C). The cytoplasm had a fine, punctated and granular appearance with uniformly round "salt and pepper" nuclei. No mitosis was quantified. They were immunoreactive for chromogranin, synaptophysin and CD56. He did not have systemic symptoms with a normal level of urinary 5-hydroxy indole acetic acid (5HIAA). Upper abdominal computed tomography showed no enlarged lymph nodes. After therapeutic argon plasma coagulation (APC) was performed electively

Correspondence: In Cheol Hwang, Gachon University Gil Medical Center, 1198 Guwol-dong, Namdong-gu, Incheon 405-760, Republic of Korea. Email: spfe0211@gmail.com, Phone: +82-32-4608207. 
(Figure 1D and 1E), he has been free of recurrence during the 50-month of follow-up (Figure 1F).

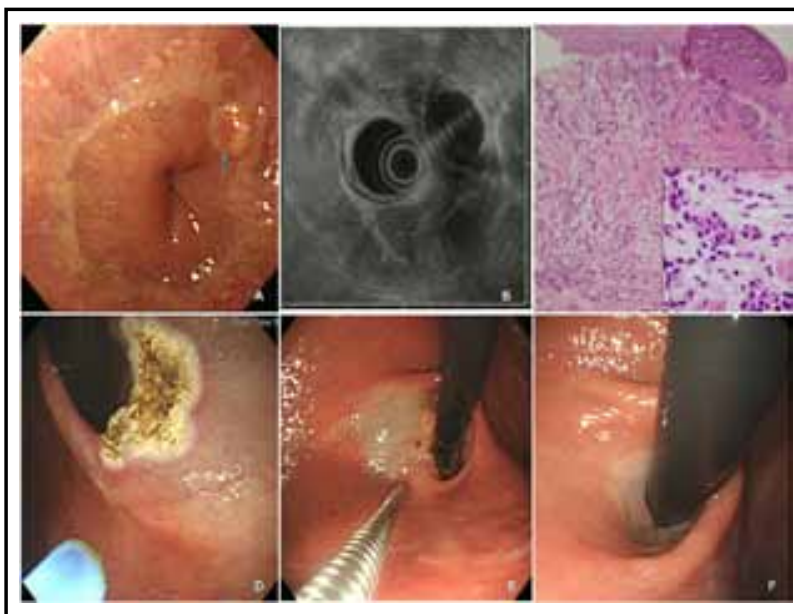

Figure 1. A: Endoscopic findings. Approximately $0.5 \mathrm{~cm}$ sized fixed elevated mass was noted at the esophagogastric junction. B: The findings of endoscopic ultrasonography. An oval shaped hypoechoic mass was observed within the submucosal layer of the esophagogastric junction. The size was $6 \mathrm{~mm}$. C: Microscopic findings of the biopsy specimen. Nests of oval shaped tumor cells have infiltrated the stroma beneath the basal layer of the esophageal epithelium (Hematoxylin and eosin stain, $\times 100)$. The high power view is shown in the inset (Hematoxylin and eosin stain, $\times 400$ ). $D$ and $E$ : Argon plasma coagulation. F: No residual tumor is found at follow-up endoscopy.

\section{DISCUSSION}

Increased endoscopic surveillance and enhanced evaluation of biopsies lead to the early detection of malignancies. Familiarity with CTs of unusual locations, such as our case, will help clinicians to prevent from missing them and to initiate appropriate diagnostic evaluation. Although most CTs develop in the gastrointestinal tract, they are rarely found in the esophagus. In a large series of 8,305 CTs of various sites, only three cases were reported, representing $0.05 \%$ of all gastrointestinal CTs and $0.02 \%$ of all esophageal cancer. ${ }^{4}$

Regrettably, knowledge about esophageal CT is primarily based on case reports which have emphasized on their poor prognosis. ${ }^{3}$ There has been a consensus that esophageal CTs are deeply invasive with high mitotic activity, ${ }^{2}$ but the histology was not considered in their review; those CTs actually might be another histologic type (e.g., small cell carcinoma showing very poor prognosis). Quite the contrary, recent reports suggest that esophageal CTs are not particularly associated with a poor prognosis, which are comparable to our case. ${ }^{5,6}$

The management of esophageal CTs has not been settled as well. In case of the CTs in other gastrointestinal tract, the treatment strategies have been determined based on size, depth of invasion, number of lesions and histological grading: for well-differentiated tumors which are smaller than $1 \mathrm{~cm}$ or fewer than 5 in number, with no extension outside the submucosal, regular endoscopic surveillance or endoscopic resection is appropriate. ${ }^{7,8}$

We performed endoscopic ablation therapy because our initial biopsy was nearly equal for the excision. Instead, we decided to undergo regular gastroscopic surveillance with measuring urinary 5-HIAA levels. APC is safe with uncommon complications and has been used for the eradication of early-stage malignancy. ${ }^{9,10}$ Recently, Nomura et al. conducted APC treatment successfully on patients with superficial carcinoma in esophagus. ${ }^{11}$

Our report attempts to make clinicians familiarize with rare CTs and to inform readers about the uncertainty of prognosis and/or treatment for esophageal CTs. We also carefully suggest that endoscopic ablation therapy may be advisable for treating localized esophageal CT, albeit in one limited case.

\section{Conflict of Interest: None.}

\section{REFERENCES}

1. Tateishi R, Taniguchi H, Wada A, Horai T, Taniguchi K. Argyrophil cells and melanocytes in esophageal mucosa. Arch Pathol. 1974; 98(2): 87-9.

2. Bosman FT, World Health Organization., International Agency for Research on Cancer. WHO classification of tumours of the digestive system. 4th ed. Lyon: International Agency for Research on Cancer; 2010:417 p.

3. Ready AR, Soul JO, Newman J, Matthews HR. Malignant carcinoid tumour of the oesophagus. Thorax. 1989; 44(7): 594-6.
4. Modlin IM, Sandor A. An analysis of 8305 cases of carcinoid tumors. Cancer. 1997; 79(4): 813-29.

5. Chuah SK, Hu TH, Kuo CM, et al. Upper gastrointestinal carcinoid tumors incidentally found by endoscopic examinations. World J Gastroenterol. 2005; 11(44): 7028-32.

6. Hoang MP, Hobbs CM, Sobin LH, Albores-Saavedra J. Carcinoid tumor of the esophagus: a clinicopathologic study of four cases. Am J Surg Pathol. 2002; 26(4): 517-22. 
7. Valentino J, Evers BM. Recent advances in the diagnosis and treatment of gastrointestinal carcinoids. Adv Surg. 2011; 45: 285-300.

8. Zhang L, Ozao J, Warner R, Divino C. Review of the pathogenesis, diagnosis, and management of type I gastric carcinoid tumor. World J Surg. 2011; 35(8): 1879-1886.

9. Barr H. Ablative mucosectomy is the procedure of choice to prevent Barrett's cancer. Gut. 2003; 52(1): 14-5.
10. Van Laethem JL, Jagodzinski R, Peny MO, Cremer M, Deviere J. Argon plasma coagulation in the treatment of Barrett's high-grade dysplasia and in situ adenocarcinoma. Endoscopy. 2001; 33(3): 257-61.

11. Nomura T, Miyashita M, Makino H, et al. Argon plasma coagulation for the treatment of superficial esophageal carcinoma. J Nippon Med Sch. 2007; 74(2): 163-7. 